\title{
GDNF gene-modified mesenchymal stem cells promote functional recovery in the rat traumatic brain injury model
}

\author{
Shiu-Min Cheng ${ }^{1}$, Ming-Hong Tai ${ }^{2}$, Shiau-Min Hwang ${ }^{3}$, Fu-Chou Cheng ${ }^{1}$ \\ ${ }^{1}$ Stem Cell Center, Department of Medical Research, Taichung Veterans General Hospital, Taichung; ${ }^{2}$ Department of Medical \\ Research, Kaohsiung Veterans General Hospital, Kaohsiung; ${ }^{3}$ Bioresources Collection and Research Center, Food Industry \\ Research and Development Institute, Hsinchu
}

Traumatic brain injury (TBI) is the leading cause of death and permanent disabled, affecting the young and adults in the most productive years of their lives. Recently, human amniotic fluid mesenchymal stem cells (hAFMSC) were reported to ameliorate functional deficits after sciatic nerve injury in rats, with some of this improvement possibly resulting from the action of cytokines secreted by these cells. Adenovirus-glial derived neurotrophic factor (Ad-GDNF) can preserve neuronal fibers and promote locomotor recovery on rats subjected to spinal cord contusion. Thus, we transfected hAFMSCs with the Ad-GDNF and investigated whether these cells contributed to improved functional recovery in TBI rats. The hAFMSCs were plate in $5 \mathrm{ml}$ of -MEM supplemented with $20 \% \mathrm{FBS}$ in a T75-flask and incubated at $37{ }^{\circ} \mathrm{C}$ with 5\% humidified $\mathrm{CO}_{2}$. The Ad-GDNF and Ad-green fluorescent protein (Ad-GFP) were prepared. The hAFMSCs were infected with Ad-GFP and Ad-GDNF. A Dragonfly fluid percussion device was used to induce a lateral fluid percussion injury model of moderate TBI in adult SD rats. The GDNF gene-modified hAFMSCs $\left(1 \times 10^{6}\right)$ were injected into the right dorsolateral striatum $4 \mathrm{~mm}$ and lateral $3 \mathrm{~mm}$ to bregma over a 2.5 -min period. Motor function recovery experiment was assayed using a rotarod test. Our preliminary findings are (1) the Ad-GFP transfected hAFMSCs after 3 days expressed the florescence in vitro, (2) 7 days after transplantation, the GFP and CM-Dil positive cells exhibited in the subventricle zone-ofactory bulb neural regeneration tract, the MSC-GDNF treatment group showed better motor behavioral recovery from 3 days through 14 days than control group, and (4) the improved effect can prolong to 28 days after transplantation. These preliminary data suggest that hAFMSCs transfected with the GDNF gene may be useful in the treatment of brain trauma and may represent a new strategy for the treatment of TBI.

Keywords: GDNF, mesenchymal stem cell, traumatic brain injury

Cell Research (2008) 18:s55. doi: 10.1038/cr.2008.145; published online 4 August 2008

Correspondence: Fu-Chou Cheng

E-mail: vc1035@gmail.com 